\title{
围绕 SDSL 的教学模式及应用
}

\author{
缪素芬 刘芳 颜素容丸 王耘丸 \\ 北京中医药大学中药学院 \\ DOI:10.32629/fcmr.v2i1.1541
}

\begin{abstract}
[摘 要] 在教学过程中,教师不仅要重视对学生知识的传授,而且更要重视教学过程对学生能力的培养 和促进得到全面的发展。本文从问题驱动、角色转换、学习形式、材料组织、思维创新和组织方法六 个维度考虑问题,提出了包含SDSL(Six-Dimension-Space Learning)教学模式, 该模式包含问题式学习、自 主学习、体验式学习、案例式学习、原点式学习和团队学习六种方法。实践证明, 该教学模式能促进学 生全面发展、提升创新能力和实践能力和自主学习能力,达到培养高素质应用型人才的培养目标。
\end{abstract}

[关键词] SDSL教学模式; 自主学习; 体验式学习; 原点式学习

中图分类号: G42 文献标识码: A

\section{Around SDSL teaching mode and application \\ Sufen Miao Fang Liu Surong Yan* Yun Wang^}

School of Traditional Chinese Medicine,Beijing University of Chinese Medicine

[Abstract] In the process of teaching, teachers should not only pay attention to the imparting of students' knowledge, but also pay attention to the cultivation of students' ability in the teaching process and promote their all-round development. From the perspectives of drive, role, form, material, thought and organization, this paper proposes SDSL (six-dimensional-space Learning) teaching model, which includes Six methods: problem Learning, independent Learning, experiential Learning, case Learning, origin Learning and team Learning. It has been proved by practice that this teaching mode can promote the comprehensive development of students, enhance their innovation ability, practical ability and independent learning ability, and achieve the goal of cultivating high-quality application-oriented talents.

[Key words] SDSL teaching mode;Independent Learning;Experiential learning; The origin learning

课堂教学以知识的传授和独立思考 能力的培养为宗旨, 因此提高课堂效率、 优化思维模式, 是教学质量的保障。传统 教学模式体现的是LBL (Lecture-Based Learning), 是各大高校一直采用的主 要教学模式, 学生在课堂上只能是被 动地学习, 一味地接受, 缺少独立思考 的过程。虽然也能达到传授知识的目 的, 但不利于学生综合素质的提高, 同 时不能提高学生的主观能动性, 忽视 了学生创新能力、独立解决问题能力 的培养, 导致教学与社会实践脱节。而 这些能力正是教学所应培养的的重点, 培养思想品德高尚、理论基础扎实、 实践能力强, 具有探索创新精神的大 学生, 是当前高等教育面临的首要任
务。当前的教学模式不能满足新时期 人才培养的需要 ${ }^{[1]}$ 。

在长期的教学实践中, 我们结合学生 的年龄特点和学习规律提出了SDSL学习 方法 (Six-Dimension-Space Learning), 并围绕SDSL构建教学模式, 简称SDSL教 学模式。SDSL是教师主导下学生自主学 习的学习方法, 该方法将问题式学习、自 主学习、体验式学习、案例式学习、原 点式学习、团队学习有效融合起来, 构成 六维空间学习方法, 故称SDSL。围绕SDSL 的教学模式旨在增强学生对学习内容的 理解程度。该教学模式下, 可以提升学生 创新能力和实践能力以及自主学习的能 力, 促进学生全面发展, 达到培养高素质 应用型人才的培养目标。

\section{SDSL学习方法与新型教学} 模式

1. 1 问题式学习

问题式学习是从驱动维度出发, 在 教学过程中教师带领学生提出问题, 分 析问题, 解决问题。带着问题去学习, 是 当今最具效率的学习方法, 它可以让学 生及时做到查缺补漏, 实现对知识的系 统掌握。问题教学法可以让学生一步步 地探索知识的奥秘, 对于提高学生学习 积极性, 促进学生全面掌握知识, 促进知 识融合与发展起到关键作用 ${ }^{[2]}$ 。

以 “问题为中心” 的教学, 是将教材 的知识点以问题的形式呈现在学生面前, 让学生在寻求和探索解决问题的思维活 动中, 掌握知识、发展智力、培养技能。 
笔者会针对这节课的教学内容来合理设 置题目, 可以让学生阶梯式地学习知识, 避免 “一堂灌” 教学模式的出现。学生 通过对问题的解决达到对知识的掌握, 更好地理解和掌握知识点, 达到学生对 知识的融会贯通, 从而促进其更好地掌 握知识点。

另外, 在课堂教学中, 还要鼓励学生 及时将不理解的问题抛出, 让大家在课 堂上去解决, 促进学生更好地理解和掌 握知识, 提高学生在教学过程中的参与 度, 进而提升教学效果。并且能激活学生 思维, 培养探索精神, 使学生掌握发现和 探索知识的方法, 提高其自学能力、思考 能力和解决问题的能力。

\section{2 自主学习}

自主学习是从角色维度强调学生在 教学过程中的主体地位。孟子曰: “君子 深造之以道, 欲其自得之也。自得之, 则 居之安; 居之安, 则资之深; 资之深, 则 取之左右逢其原, 故君子欲其自得之 也”。他强调的是学习贵在自得。老师讲 的, 是老师的。学习就要把老师讲的知识 内化为自己的东西, 用起来才能左右逢 源。那么, 在学习方法上, 就不能采用“满 堂灌”的教学方式, 学生被动机械地接受 知识, 没有内化的过程。一个人获得高深 的造诣, 要靠自己积极主动的学习; 经过 积极主动的学习, 所学的知识就能牢固 地掌握, 从而积累起丰富的知识, 在应用 知识的时候才能够得心应手。所以需要 培养学生自主学习的能力。

自主学习是以学生作为学习的主体, 通过学生独立地分析、探索、实践、质 疑、创造等方法来实现学习目标。倡导 学生主动参与、乐于探究、勤于动手, 培养学生搜集和处理信息的能力、获取 新知识的能力、分析和解决问题的能力 以及交流与合作的能力 ${ }^{[3]}$ 。

培养学生的自主学习能力是素质教 育的要求, 也是人的全面发展和 21 世纪 的需要。培养自主学习能力不仅能优化 课堂教学, 提高教学效率, 而且有利于学 生今后的学习。自主学习能力是学生终 身成长和发展进步的基础, 是学生在校 学习以及在社会发展的必备能力, 自主
学习能力的培养已成为当今高等教育的 重中之重。

培养学生的自主学习能力要以学生 为本位, 在学生积极参与的学习过程中 培养和提高。我们首先应该加强学生的 自主学习意识; 其次应该渗透培养学生 自主学习所必备的各种能力; 最后教师 要优化教学环境, 鼓励学生自我探索, 在 课堂教学中培养学生自主学习的能力。

\section{3 体验式学习}

体验式学习是从学习形式的维度, 强调知识运用能力的培养, 而非单纯的 知识传授。体验式学习法可追溯到孔子。 孔子说: “吾听吾忘, 吾见吾记, 吾做吾 悟”。就是说, 我所听到的, 我可能会忘 记; 我所看到的, 我可能会记住; 但是我 所做过的, 我才会理解。宋代诗人陆游也 强调: “纸上得来终觉浅, 绝知此事要躬 行”, 由此可见, 只有亲身体验的事物, 才是最可靠、最牢固的。

体验式学习法是指通过实践和体验 来认知知识或事物, 或者说通过体验能 使学习者完完全全地参与学习过程, 使 学习者真正成为课堂的主角。在体验式 学习过程中, 知识、技能的获得, 情感、 态度的形成, 都是以体验活动为基础的。 教师的作用不再是一味地单方面传授知 识, 而是为学生做好体验开始的准备工 作, 让学生全身心地投入学习过程。体验 式学习像生活中其他任何一种体验一样, 是内在的, 是个人参与所得。正因为全身 心的参与, 从而使得学习效率、知识理 解、知识记忆持久度都大幅度提升。

\section{4 案例式学习}

案例式学习是从材料组织的维度, 强调知识点的融合与应用, 培养学生知 识融会贯通能力和知识应用能力的培 养。案例教学是将案例运用到理论教学 中, 是一种开放式、互动式的新型教学方 式。通常, 案例教学要经过事先周密的策 划和准备, 要使用特定的案例并指导学 生提前阅读, 然后组织学生以案例为中 心, 展开讨论, 对问题进行具体分析, 总 结出知识要点, 通过各种信息、知识、经 验、观点的碰撞来达到启示理论和启迪 思维的目的。最后教师进行总结知识点
并进行升华 ${ }^{[4]}$ 。

案例教学法是目前能将课本和实际 经验两者很好的结合起来的一种重要而 有效的教学方法。案例学习强调 “通过 别人的教训, 增长自己的智慧”。通过收 集的案例进行分析, 不仅学会知识, 而且 可以举一反三, 提高知识应用能力。

\section{5 原点式学习}

原点式学习是从思维创新维度, 强 调知识是科学家发现的, 回归科学家的 历程, 将学习过程和科学家发现知识的 过程融合在一起。教材记述的是前人探 索的结果, 一般不涉及探索、思考的过程, 但过程比结果更重要, 教学的关键和难 处正是在于揭示前人的发现过程和思想 脉络。强调 “知识不是学来的, 而是想出 来的”。在知识不存在的情况下, 人类会 怎么考虑? 在思考的过程中, 再现前人 发现知识的过程, 为什么提出这个概念, 主要解决什么问题? 把学习知识的过程, 变成了学生自主探究的 “再发现”、“再 创造”的过程, 使学生在原点式学习的环 节中, 学会如何思考问题, 培养科研思路, 提高学生各种能力, 包括质疑能力。激发 学生对专业的兴趣和对知识的探索欲。 变有限的基础知识为无限的创新才能, 更好的适应社会的发展。

\section{6 团队学习}

团队学习是指为了实现学习目标, 有计划有组织开展的两人或两人以上学 生参与的群体性学习活动。团队学习是 从组织的维度, 强调协同学习的重要性, 注重学生团队协作能力的培养。

在高校教育的主要内容中, 专业知 识的传授是重要的一部分。然而, 教育更 本质、更核心的内容应该是激发学生学 习的主动性和创新能力及学生综合素质 的培养。而团队学习融入教学中, 具有其 他教学模式不可比拟的优点, 特别是在 学生能力提高和综合素质培养方面更加 突出 ${ }^{[5-6]}$ 。

首先, 团队学习能够促进学习交流, 形成头脑风暴, 拓宽学生的学习思路。 “三个臭皮匠, 顶个诸葛亮”。团队学习 就是把个人掌握的知识、思想与方法、 经验与教训等, 在团队中共享, 能发挥团 
队成员的集体智慧, 让团队智慧大于单 一个人智慧的总和, 让学习成为现实的 创造力。

其次在教师的指导下, 团队成员能 够在课堂内外借助一切可以学习的信息 资源, 解决教师布置的实际问题。良好的 团队学习氛围, 不仅需要个人的努力, 更 需要团队间每个队员的密切配合, 可以 加强同学间的交流与配合, 发挥团队协 作精神, 变被动学习为主动学习, 刻苦钻 研。团队学习过程中能培养学生的责任 意识, 学生不仅要对自己负责, 而且还要 对团队负责, 对社会负责, 进而培养学生 的社会责任感。

\section{SDSL学习法的融合与实践}

SDSL的六种学习方法不是孤立的, 而是一个完整的有机整体, 六种学习方 法各从不同的维度组织学习与教学 (问 题式学习是从驱动维度; 自主学习从角 色维度; 体验式学习从形式维度; 案例 式学习从材料维度; 原点式学习从思维 维度; 团队学习从组织维度)。在学生学 习过程中, 并不是每个知识点的学习都 要生搬硬套的均衡运用 “六维教学法” 中的所有方法, 而是根据具体知识点的 特点, 采用的学习方法可有所侧重。

北京中医药大学不断深化教育教 学改革, 为培养具有中医药创新能力的 复合型人才进行着不解的努力。笔者所 在教研室承担《医药高等数学》和《统 计学基础》两门课程, 《医药高等数学》 和《统计学基础》的教学应该在注重数 学思想教育和统计思想方法的基础上,
加强培养应用数学解决实际问题的能 力。在这两门课程的教学中, 均采用了 SDSL (Six-Dimension-Space Learning) 教学模式, 取得了良好的教学效果。

在《医药高等数学》教学过程中, 主要针对《医药高等数学》课程里在中 医药领域有广泛应用的章节, 通过设计 问题 (问题式学习), 对学生分组开展讨 论, 学生首先自己查阅资料, 认真思考 (自主学习), 然后再小组讨论 (团队学 习), 引导学生深入理解数学相关概念的 产生背景 (原点式学习), 培养中医药学 生具有理性分析的中医药思维方式, 并 能熟练运用到今后的中医药专业问题 (体验式学习)。使得学生在整个课程学 习过程中, 感受到数学的学习不再是枯 燥的, 在了解知识的来龙去脉的同时, 整 体素质也得到了全面的发展。

在《统计学基础》课程中, 将课程分 为两大部分, 一是原理部分, 包括基础 的、经典的、与专业密切相关的统计方 法及软件实现; 二是应用部分, 针对具体 的统计方法, 增加学生应用实践环节, 其 内容包含多个专题。在各个部分中, 我们 均采用了六维空间学习法, 针对具体的 学习内容, 综合采用问题式学习、自主学 习、体验式学习、案例式学习、原点式 学习、团队学习的组织方式, 以中药研究 中的具体专业问题为导向, 引导学生实 现医药专业与统计方法的有机融合, 取 得了很好的效果。

\section{3 结语}

实践证明在教学中实施 “六维空间
教学法”, 改变了课堂的教学面貌, 学生 在教师的引导下主动探究, 积极地参与 到课堂教学过程中。在教学过程中, 笔者 也体会到, 正所谓“教须有法, 但无定法”, 并不是每门课程的每节课都要生搬硬套 的去运用 “六维空间教学法” 中的所有 方法, 应该结合讲授的内容及该章节的 教学目标, 并在教学实践选择合适的教 学方法, 才能切实地提高教学质量, 达到 教学效果。

\section{[参考文献]}

[1]刘炎.基于能力本位的高等数学 课堂教学模式的改革和探究 [J]. 才 智,2018,(36):63-64.

[2]孙兆芬,郭建华.问题教学法视 角下的数学教学——以 “平均变化 率” 为例 [J]. 中学数学研究(华南师范大 学版),2017,(18):29-31+18.

[3]王正昂,李婉.互联网视角下大学 生高等数学自主学习研究[J].西部素质 教育,2017,3(13):209-210.

[4]李培.高等数学案例教学法 的应用实践研究 [J].湖北函授大学学 报,2018,(1):171-172.

[5]张景川, 楚合营, 黄新成. 基于团 队学习的大学物理教学模式探究与实践 [J].大学教育,2014,(17):97-98.

[6]李恒凯,李子阳,李芹.面向创新 能力培养的本科生团队学习模式一 一以GIS专业为例 $[J]$. 上绕师范学院学 报,2013,36(8):74-78. 